\title{
Delayed Antiwindup Control Using a Decoupling Structure
}

\author{
Huawei Zhu, ${ }^{1}$ Guozheng Qin, ${ }^{1}$ Yingxin Yan, ${ }^{2}$ Zhichao Jiang, ${ }^{2}$ and Zhisheng Duan ${ }^{1}$ \\ ${ }^{1}$ State Key Lab for Turbulence and Complex Systems, Department of Mechanics and Aerospace Engineering, \\ College of Engineering, Peking University, Beijing 100871, China \\ ${ }^{2}$ Beijing Institute of Near-Space Vehicle's System Engineering, Beijing 100076, China
}

Correspondence should be addressed to Zhisheng Duan; duanzs@pku.edu.cn

Received 28 March 2013; Accepted 29 April 2013

Academic Editor: Guanghui Wen

Copyright (C) 2013 Huawei Zhu et al. This is an open access article distributed under the Creative Commons Attribution License, which permits unrestricted use, distribution, and reproduction in any medium, provided the original work is properly cited.

\begin{abstract}
This paper investigates the antiwindup (AW) control problem for plants with input saturation. The AW compensator is not activated as soon as input saturation occurs as usual. A delayed decoupling structure is first proposed. Then, appropriate linear matrix inequalities (LMIs) are developed to determine a plant-order AW compensator. Effectiveness of the presented AW technique is illustrated by a fighter aircraft model.
\end{abstract}

\section{Introduction}

Input saturation is one of the most frequently encountered nonlinearities in control design for linear plants. Most controllers are designed assuming that the inputs have no limits. If input saturation appears, output performance often deteriorates. Slight performance degradation could be large overshoot or sluggish response. Severe performance deterioration could be persistent oscillation, sometimes even instability.

Multiagent control has become a hot topic of academic research in recent years. This is because it can be used in various fields, such as physics, biology, industry, and military applications. The two main applications of multiagent control in industry and military are the multirobot cooperative control and the multivehicle flight control, respectively. Although these two applications have been intensively studied, however, few results consider the case when actuators are limited [1]. The well-known AW control is one of the most successful methodologies applied in practice to deal with plants with input saturation. As summed in $[2,3]$, the essential idea of AW control is adding control modification to a nominal linear controller. The nominal linear controller is first synthesized for the plant ignoring saturation, and then an AW compensator is designed to improve output performance when saturation occurs. Sufficient conditions to design a static AW compensator were provided in [4] in terms of LMIs. Necessary and sufficient conditions for the existence of a plant-order AW compensator were given in [5]. A novel, less conservative sector condition was presented in [6], and a static AW compensator for both stable and unstable plants was obtained therein. A switching AW design using multiple Lyapunov functions was proposed in [7]. In $[4,5]$, attention was focused on minimizing the influence of a generic disturbance signal $w$ in the output. In $[6,7]$, concern was on maximization of the region of attraction. Innovatively in [8], Weston and Postlethwaite proposed a structure, which established a specific formulation of the error between the plant outputs with and without input saturation. Then, in [9], two different AW compensators were designed to make the error as small as possible based on the structure.

Usually, the AW compensator was activated as soon as input saturation occurs. However, a static AW compensator designed in [10] was not activated immediately but belatedly. It was found in [10] that delayed activation of AW compensator can lead to a better performance compared with the immediate one. In order to improve AW control performance and derive a simpler LMI condition for the AW compensator construction, motivated by the structure proposed in [8], a modified delayed decoupling structure is developed in this paper. Then, sufficient conditions in terms of LMIs are obtained to minimize the error between the plant outputs with and without input saturation. 


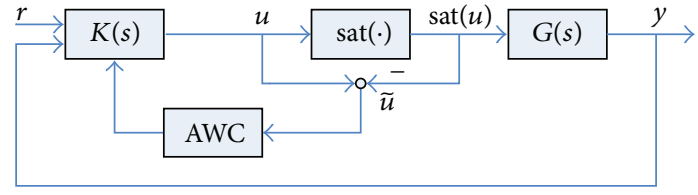

FIGURE 1: General structure of AW control.

The remainder of this paper is organized as follows. In Section 2, the structure, which has a decoupling feature, is introduced. Main results of this paper are presented in Section 3. A new modified delayed AW decoupling structure is first proposed. And the AW compensator is constructed for single-input plants by making a set of LMIs feasible. It is also extended to multi-input plants. A fighter craft model is used to illustrate the proposed AW technique. Conclusions are given in Section 4.

\section{A Decoupling Structure}

A general AW control structure is shown in Figure 1, where $G(s)$ is the plant, $K(s)$ is the nominal linear controller, sat(·) is the input saturation function, and block AWC is the AW compensator. $G(s)$ is a stable linear plant subject to input saturation as

$$
G(s) \sim\left\{\begin{array}{c}
\dot{x}_{p}=A_{p} x_{p}+B_{p} \text { sat }(u), \\
y=C_{p} x_{p}+D_{p} \text { sat }(u),
\end{array}\right.
$$

where $\left(A_{p}, B_{p}\right)$ is stabilizable, $x_{p} \in \mathbb{R}^{n}, y \in \mathbb{R}^{q}, u \in \mathbb{R}^{p}$, and $\operatorname{sat}(u)$ is the saturated input. The saturation function sat $(\cdot)$ satisfies $\operatorname{sat}(u)=\left[\operatorname{sat}\left(u_{1}\right), \operatorname{sat}\left(u_{2}\right), \ldots, \operatorname{sat}\left(u_{p}\right)\right]^{T}, \operatorname{sat}\left(u_{i}\right)=$ $\operatorname{sign}\left(u_{i}\right) \min \left(\left|u_{i}\right|, \bar{u}_{i}\right) \cdot \bar{u}_{i}$ is the amplitude limit, $\bar{u}_{i}>0, i=$ $1,2, \ldots, p$. The state-space descriptions of $K(s)$ and AW compensator are, respectively

$$
\begin{gathered}
K(s) \sim\left\{\begin{array}{l}
\dot{x}_{c}=A_{c} x_{c}+B_{c} e, \\
u=C_{c} x_{c}+D_{c} e,
\end{array}\right. \\
\text { AWC } \sim\left\{\begin{array}{l}
\dot{x}_{\mathrm{aw}}=A_{\mathrm{aw}} x_{\mathrm{aw}}+B_{\mathrm{aw}} \tilde{u}, \\
\eta=\left(\eta_{1}^{T}, \eta_{2}^{T}\right)^{T}=C_{\mathrm{aw}} x_{\mathrm{aw}}+D_{\mathrm{aw}} \tilde{u},
\end{array}\right.
\end{gathered}
$$

where $\tilde{u}:=u-\operatorname{sat}(u)$ is the error between the unsaturated input and saturated input, $e:=r-y$ is the tracking error, and $r$ is the reference to be tracked. The AW compensator is activated only when input saturation occurs. As mentioned earlier, the essential idea of AW control is adding control modification to $K(s)$. The state and output equations of $K(s)$ are modified by the AWC's output $\eta=\left(\eta_{1}^{T}, \eta_{2}^{T}\right)^{T}$ as

$$
\begin{gathered}
\dot{x}_{c}=A_{c} x_{c}+B_{c} e+\eta_{1}, \\
u=C_{c} x_{c}+D_{c} e+\eta_{2} .
\end{gathered}
$$

An appealing AW structure, which is a special case of the general structure, proposed by Weston and Postlethwaite in [8], is shown in Figure 2(a), where the design of AW compensator turns into the conditioning of a transfer function $M(s)$. With all signals labeled the same, the structure in Figure 2(a) can be equivalently redrawn as in Figure 2(b). Note that in Figure 2(b), a dead-zone function $d z(\cdot)$ is introduced, which maps the unsaturated input $u$ to the input error $\tilde{u}$; that is, $d z(u)=\widetilde{u}$. If $u \in \mathscr{U}$, where $\mathscr{U} \in \mathbb{R}^{p}$ is a compact set defined as $\mathscr{U}=\left[-\bar{u}_{1}, \bar{u}_{1}\right] \times\left[-\bar{u}_{2}, \bar{u}_{2}\right] \times \cdots \times\left[-\bar{u}_{p}, \bar{u}_{p}\right]$, then $d z(u)=0$. What is fascinating about the structure in Figure 2(b) is that it can be seen as two decoupled subsystems: the nominal linear closed-loop system (NLCS) and the nonlinear path. If NLCS and the nonlinear path are stable, respectively, the stability of the overall system in Figure 2(b) follows. The NLCS is stable, as it is guaranteed by $K(s)$. The NLCS's output $y_{\text {lin }}$ is what one tries to recover when input saturation occurs. Thus, the AW compensator should be designed to ensure stability of the nonlinear path, further on, if possible, to make the output difference $y_{d}=y_{\text {lin }}-y$ as small as possible.

One selection for $M(s)$ in Figure 2(a) is a right coprime factor of $G(s)$; that is, $G(s)=N(s) M^{-1}(s)$. The state space descriptions of $M(s)$ and $N(s)$ can be chosen as

$$
\left(\begin{array}{c}
M(s) \\
N(s)
\end{array}\right) \sim \begin{array}{c|c}
A_{p}+B_{p} F & B_{p} \\
\hline F & I \\
C_{p}+D_{p} F & D_{p}
\end{array} .
$$

The conditioning with $M(s)$ turns into the design of matrix F. $G(s) M(s)=N(s)$, and the state space description of AW compensator is

$$
\begin{gathered}
\dot{x}_{\mathrm{aw}}=\left(A_{p}+B_{p} F\right) x_{\mathrm{aw}}+B_{p} \tilde{u}, \\
u_{d}=F x_{\mathrm{aw}}, \\
y_{d}=\left(C_{p}+D_{p} F\right) x_{\mathrm{aw}}+D_{p} \tilde{u} .
\end{gathered}
$$

Remark 1. Note that all the initial states and the control inputs of $M(s)$ and $N(s)$ are equal. Therefore the states of $M(s)$ and $N(s)$ are identical. Under this circumstance, although $M(s)$ and $N(s)$ are different transfer functions, they can still share the same state $x_{\mathrm{aw}}$.

Define the distance between a vector $x$ and a compact set $\mathcal{S}$ as $\operatorname{dist}(x, \mathcal{S}):=\inf _{y \in \mathcal{S}}\|x-y\|$. Then, the objective of this paper, which is based on the decoupling AW structure proposed in [8], is to solve the AW control problem defined as follows.

Definition 2 (see [9]). The AW compensator (5) is said to solve the AW control problem for plant (1) if the closedloop system in Figure 2(a) is well posed and the following conditions hold.

(1) If $\operatorname{dist}\left(u_{\operatorname{lin}}, \mathcal{U}\right)=0$ for all $t \geq 0$, then $y_{d}=0$ for all $t \geq 0$ (assuming zero initial condition for AW compensator).

(2) If $\operatorname{dist}\left(u_{\text {lin }}, \mathscr{U}\right) \in \mathscr{L}_{2}$, then $y_{d} \in \mathscr{L}_{2}$.

The AW compensator (5) is said to solve the strong AW control problem if in addition, the following condition is satisfied.

(3) The operator $\tau: u_{\text {lin }} \mapsto y_{d}$ is well defined and the $\mathscr{L}_{2}$ gain from $u_{\text {lin }}$ to $y_{d}$ is less than $\gamma$; namely, $\left\|y_{d}\right\|_{2}<$ $\gamma\left\|u_{\text {lin }}\right\|_{2}$. 


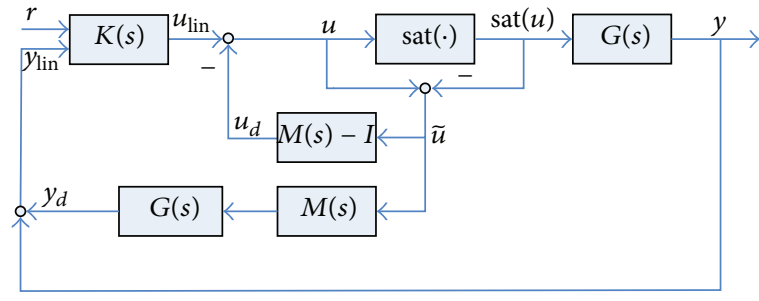

(a) Conditioning with $M(s)$



(b) An equivalent decoupling structure

Figure 2: A decoupling structure.

Remark 3. Note that condition 3 in Definition 2 implies condition 2. In this paper, only the strong AW control problem is considered.

\section{Main Results}

Immediate AW compensation may increase safety of the overall system, being away from instability, for example, but the performance may be no better than leaving the controller uncompensated during some moderate input saturation, as the numerical example demonstrated in [10]. If the nominal controller is robust, the system still demonstrates good performance during this modest saturation stage. Thus, the delayed activation of AW compensation is considered.

3.1. Delayed Decoupling AW Structure. To realize certain delay in activating the AW compensator, an artificial saturation function is added after the controller, and thus the structure in Figure 2(a) becomes as in Figure 3. This structure has two saturation nonlinearities, and thus it is not equivalent to the decoupling structure in Figure 2(b). In order to sustain a decoupling feature, Figure 3 needs to be modified. A new decoupling structure with delayed saturation is therefore presented in Figure 4(a). Note that a time-varying gain $G_{d}=\operatorname{diag}\left[g_{1}(t), g_{2}(t), \ldots, g_{p}(t)\right]$ is introduced in Figure 4(a), where $g_{i}(t)$ is defined as

$$
g_{i}(t)= \begin{cases}1, & \left|u_{i}\right| \leq \bar{u}_{i}, \\ \frac{\operatorname{sgn}\left(u_{i}\right) \bar{u}_{i}}{u_{i}}, & \bar{u}_{i}<\left|\bar{u}_{i}\right|<\frac{\bar{u}_{i}}{g_{d}}, \\ g_{d}, & \left|u_{i}\right| \geq \frac{\bar{u}_{i}}{g_{d}} .\end{cases}
$$

Here $g_{d}$ is the delayed saturation index, $0<g_{d}<1$. The AW compensator is activated when the input magnitude is higher than the delayed saturation level $\bar{u}_{i} / g_{d}$, rather than $\bar{u}_{i}$. Now treat $\widehat{G}(s):=G(s) G_{d}$ as a new plant, which is linear parameter-varying (LPV). The time varying matrix gain $G_{d}$ is continuous in the known bounds, $G_{d} \in\left[g_{d}, 1\right]$. Since the expressions for $u, y, \widetilde{u}$ are the same in Figures 4(a) and 4(b), the two figures are mathematically equivalent if the following equation holds:

$$
\widehat{G} u_{\operatorname{lin}}=\widehat{N} \tilde{u}+\widehat{G}\left(u_{\operatorname{lin}}-\widehat{M} \tilde{u}\right) .
$$

Taking the same steps as in [11], where the decoupling AW structure is extended to LPV system, the equivalence between

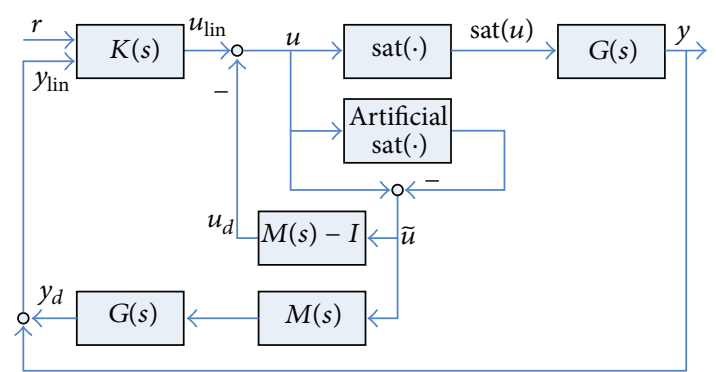

FIGURE 3: Conditioning with $M(s)$ (delayed).

Figures 4(a) and 4(b) follows. Note that in the nonlinear path, $\widehat{G}(s)$ replaces the original $G(s)$. Therefore, the state space description of the AW compensator becomes

$$
\begin{gathered}
\dot{x}_{\mathrm{aw}}=\left(A_{p}+B_{p} G_{d} F\right) x_{\mathrm{aw}}+B_{p} G_{d} \tilde{u}, \\
u_{d}=F x_{\mathrm{aw}}, \\
y_{d}=\left(C_{p}+D_{p} G_{d} F\right) x_{\mathrm{aw}}+D_{p} G_{d} \tilde{u} .
\end{gathered}
$$

3.2. Delayed AW Synthesis Using LMI. In order to analyze the behavior of the new system, for intuitiveness, single-input $(p=1)$ plants are considered first. And one has the following theorem.

Theorem 4. For a single-input saturated plant, the strong $A W$ control problem in Definition 2 is solved if there exist a symmetric positive definite matrix $Q \in \mathbb{R}^{n \times n}$, a diagonal positive definite matrix $S \in \mathbb{R}^{1 \times 1}$, a matrix $R \in \mathbb{R}^{1 \times n}$, and a positive real scalar $\gamma$ such that

$$
\left(\begin{array}{ccc}
A_{p} Q+Q A_{p}^{T}+B_{p} G_{d} R+R^{T} G_{d} B_{p}^{T} & * & * \\
0 & -\gamma I & * \\
C_{p} Q+D_{p} G_{d} R & 0 & -\gamma I
\end{array}\right)<0
$$

for $G_{d}=1$ and

$$
\left(\begin{array}{cccc}
A_{p} Q+Q A_{p}^{T}+B_{p} G_{d} R+R^{T} G_{d} B_{p}^{T} & * & * & * \\
0 & -\gamma I & * & * \\
C_{p} Q+D_{p} G_{d} R & 0 & -\gamma I & * \\
S G_{d} B_{p}^{T}-R & I & \left(D_{p} G_{d} S\right)^{T} & -2 S
\end{array}\right)<0
$$




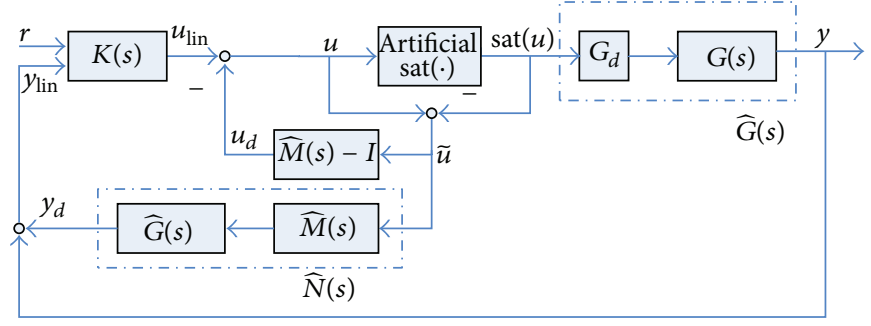

(a) Conditioning with $M(s)$ (modified)

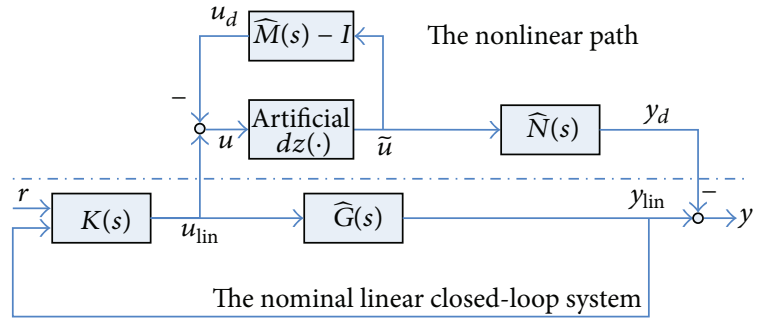

(b) The equivalent decoupling structure (modified)

FIgURE 4: The delayed decoupling structure.

for $G_{d}=g_{d}$. Furthermore, the designing matrix $F$ for $A W$ compensator (8) to guarantee $\mathscr{L}_{2}$ performance requirement $\left\|y_{d}\right\|_{2}<\gamma\left\|u_{\text {lin }}\right\|_{2}$ is given by $F=R Q^{-1}$.

Proof. The operation of the saturated plant with delayed activation of AW compensator can be separated into two stages.

Stage $1\left(|u| \leq \bar{u} / g_{d}\right)$. The input is below the artificial saturation level. The input to AW compensator is zero, namely, $\widetilde{u}=0$, and $G_{d} \in\left[g_{d}, 1\right]$. Then, following the standard approach, if inequality

$$
\dot{V}+\gamma^{-1} y_{d}^{T} y_{d}-\gamma u_{\operatorname{lin}}^{T} u_{\operatorname{lin}}<0
$$

holds, it gives $\left\|y_{d}\right\|_{2}<\gamma\left\|u_{\text {lin }}\right\|_{2}$, and the strong AW control problem is solved. Choose the Lyapunov function in (11) as $V=x_{\text {aw }}^{T} P x_{\text {aw }}$ where $P=P^{T}>0$, and substitute (8) into inequality (11); one has

$$
\begin{aligned}
& {\left[\left(A_{p}+B_{p} G_{d} F\right) x_{\mathrm{aw}}\right]^{T} P x_{\mathrm{aw}}+x_{\mathrm{aw}}^{T} P\left[\left(A_{p}+B_{p} G_{d} F\right) x_{\mathrm{aw}}\right]} \\
& \quad+\gamma^{-1}\left[\left(C_{p}+D_{p} G_{d} F\right) x_{\mathrm{aw}}\right]^{T}\left[\left(C_{p}+D_{p} G_{d} F\right) x_{\mathrm{aw}}\right] \\
& \quad-\gamma u_{\mathrm{lin}}^{T} u_{\mathrm{lin}}<0 .
\end{aligned}
$$

Then, by Schur complement and congruency transformation, inequality (12) becomes LMI (9) in Theorem 4 with $Q=$ $P^{-1}, R=F Q$; thus, $F=R Q^{-1}$. Because $G_{d} \in\left[g_{d}, 1\right]$ at Stage 1, it suffices to check LMI (9) for $G_{d}$ at the vertices, namely for $G_{d}=g_{d}$ and $G_{d}=1$. The stability of the nonlinear path is thus ensured. Note that during this stage, the "nominal" closed-loop system is linear time-varying as shown in Figure 4(b). Leaving the nominal controller $K(s)$ undisturbed when $|u| \in\left(\bar{u}, \bar{u} / g_{d}\right]$, the stability of new NCLS is preserved by the robustness of controller $K(s)$. With the two subsystems being stable, the stability of the overall closedloop system at Stage 1 follows.

Stage $2\left(|u|>\bar{u} / g_{d}\right)$. During this stage, the controller's output exceeds the delayed saturation level $\bar{u} / g_{d}$. As a result, $\widetilde{u}$ is nonzero, the AW compensator is activated, and $G_{d}=g_{d}$. Due to $\widetilde{u}^{T} u \geq \widetilde{u}^{T} \widetilde{u}$, the sector condition $2 \widetilde{u}^{T} W(u-\widetilde{u}) \geq 0$ always holds, where $W$ is a diagonal positive definite matrix. Then, a sufficient condition for inequality (11) is

$$
\dot{V}+\gamma^{-1} y_{d}^{T} y_{d}-\gamma u_{\operatorname{lin}}^{T} u_{\operatorname{lin}}+2 \widetilde{u}^{T} W(u-\widetilde{u})<0 .
$$

Then, following the similar steps as [9], if inequality (13) holds, the strong AW control problem is solved. Substitute (8) into inequality (13); one has

$$
\begin{aligned}
{\left[\left(A_{p}\right.\right.} & \left.\left.+B_{p} G_{d} F\right) x_{\mathrm{aw}}+B_{p} G_{d} \tilde{u}\right]^{T} P x_{\mathrm{aw}} \\
& +x_{\mathrm{aw}}^{T} P\left[\left(A_{p}+B_{p} G_{d} F\right) x_{\mathrm{aw}}+B_{p} G_{d} \tilde{u}\right] \\
& +\gamma^{-1}\left[\left(C_{p}+D_{p} G_{d} F\right) x_{\mathrm{aw}}+D_{p} G_{d} \widetilde{u}\right]^{T} \\
& \times\left[\left(C_{p}+D_{p} G_{d} F\right) x_{\mathrm{aw}}+D_{p} G_{d} \tilde{u}\right] \\
& -\gamma u_{\mathrm{lin}}^{T} u_{\mathrm{lin}}+2 \widetilde{u}^{T} W\left(u_{\mathrm{lin}}-F x_{\mathrm{aw}}-\widetilde{u}\right)<0 .
\end{aligned}
$$

Inequality (14) can be rewritten as

$$
\left(\begin{array}{lll}
x_{\mathrm{aw}}^{T} & \tilde{u}^{T} & u_{\text {lin }}^{T}
\end{array}\right)\left[X-Y^{T}(-\gamma)^{-1} Y\right]\left(\begin{array}{c}
x_{\mathrm{aw}} \\
\tilde{u} \\
u_{\text {lin }}
\end{array}\right)<0,
$$

where

$$
\begin{gathered}
X=\left(\begin{array}{ccc}
\left(A_{p}+B_{p} G_{d} F\right)^{T} P+P\left(A_{p}+B_{p} G_{d} F\right) & * & * \\
B_{p}^{T} G_{d} P-W F & -2 W & * \\
0 & W & -\gamma I
\end{array}\right), \\
Y=\left(\begin{array}{lll}
C_{p}+D_{p} G_{d} F & D_{p} G_{d} & 0
\end{array}\right) .
\end{gathered}
$$

According to the standard Schur complement and exerting congruency transformation, one has LMI (10) with $Q=$ $P^{-1}, S=W^{-1}, R=F Q$; thus, $F=R Q^{-1}$. Similar to Stage 1 , with the two subsystems being stable, the stability of the overall closed-loop system at Stage 2 follows.

Note that LMI (10) for $G_{d}=g_{d}$ implies that LMI (9) for $G_{d}=g_{d}$, thus only $G_{d}=1$ is checked for LMI (9) in the theorem. And this completes the proof.

The section of the delayed saturation index $g_{d}$ can be determined by an iterative algorithm, starting at an initial 
value and stopping at a point when the $\mathscr{L}_{2}$ gain $\gamma$ is optimized. By applying the AW compensator designed using Theorem 4, the nominal linear performance is recovered as much as possible. The static and dynamic AW compensators designed in $[10,12]$ used the states $x=\left(x_{p}^{T}, x_{c}^{T}\right)^{T}$ and $x=\left(x_{p}^{T}, x_{c}^{T}, x_{\mathrm{aw}}^{T}\right)^{T}$ respectively, in the Lyapunov function for LMI derivation. In this paper, only $x_{\mathrm{aw}}$ is used, and thus the LMIs to be solved here are much simpler than the resulting LMIs in $[10,12]$.

For a multi-input plant, $G_{d}$ is no longer a scalar, but a $p \times p$ diagonal matrix. Because each diagonal element of $G_{d}$ varies between $g_{d}$ and 1 , thus $G_{d}$ can be expressed as a linear combination of the vertices as follows:

$$
\begin{array}{r}
G_{d}=\sum_{j=1}^{2^{p}} \alpha_{j} G_{d}^{j}, \quad \sum_{j=1}^{2^{p}} \alpha_{j}=1, \\
\alpha_{j} \in[0,1], G_{d}^{j} \in \mathscr{G}, j=1,2, \ldots, 2^{p},
\end{array}
$$

where $\mathscr{G}=\left\{\operatorname{diag}\left[g_{1}, g_{2}, \ldots, g_{p}\right]: g_{i}=g_{d}\right.$ or $1, i=$ $1,2, \ldots, p\}$. Let $G_{d}^{1}=\operatorname{diag}[1,1, \ldots, 1]$; similarly, one has the following theorem.

Theorem 5. For a multi-input saturated plant, the strong $A W$ control problem in Definition 2 is solved if there exist a symmetric positive definite matrix $Q \in \mathbb{R}^{n \times n}$, a diagonal positive definite matrix $S \in \mathbb{R}^{p \times p}$, a matrix $R \in \mathbb{R}^{p \times n}$, and a positive real scalar $\gamma$ such that LMI (9) holds for $G_{d}=G_{d}^{1}$ and

$$
\left(\begin{array}{cccc}
A_{p} Q+Q A_{p}^{T}+B_{p} G_{d}^{j} R+R^{T} G_{d}^{j} B_{p}^{T} & * & * & * \\
0 & -\gamma I & * & * \\
C_{p} Q+D_{p} G_{d}^{j} R & 0 & -\gamma I & * \\
S G_{d}^{j} B_{p}^{T}-R & I & \left(D_{p} G_{d}^{j} S\right)^{T} & -2 S
\end{array}\right)<0
$$

for all $j=2,3, \ldots, 2^{p}$. Furthermore, the designing matrix $F$ for $A W$ compensator (8) to guarantee $\mathscr{L}_{2}$ performance requirement $\left\|y_{d}\right\|_{2}<\gamma\left\|u_{\text {lin }}\right\|_{2}$ is given by $F=R Q^{-1}$.

Proof. The proof follows the same steps as Theorem 4, and thus it is omitted here.

3.3. Application Example. Consider a linearized fighter aircraft model (matrices $A_{p}, B_{p}$, and $C_{p}$ can be found from equations (A.2)-(A.4) in Yee et al. [13]). The aircraft has six states $(u, v, w, p, q, r)$. The first three states are airspeed in the $X$-, $Y$-, and $Z$-axes of the body frame, respectively. The last three states are roll, pitch, and yaw rates about $X_{-}, Y_{-}$, and $Z$-axes. The outputs $(\dot{\mu} \alpha \beta)$ are, respectively, stability-axis roll rate, angle-of-attack, and sideslip angle. The inputs $\left(\delta_{h}, \delta_{a}\right.$, $\delta_{r}$ ) are elevator, aileron, and rudder deflection angles, with control surface constraints $-25^{\circ} \leq \delta_{h} \leq 25^{\circ},-20^{\circ} \leq \delta_{a} \leq$ $20^{\circ}$, and $-30^{\circ} \leq \delta_{r} \leq 30^{\circ}$. The reference to be tracked is shown in Figure 5.

Without consideration of input saturation, the aircraft demonstrates precise and fast tracking response, as shown in Figure 6(a), using a continuous-time robust $\mathscr{H}_{\infty}$ controller designed in [13] (matrices $A_{c}, B_{c}, C_{c}$, and $D_{c}$ are obtained

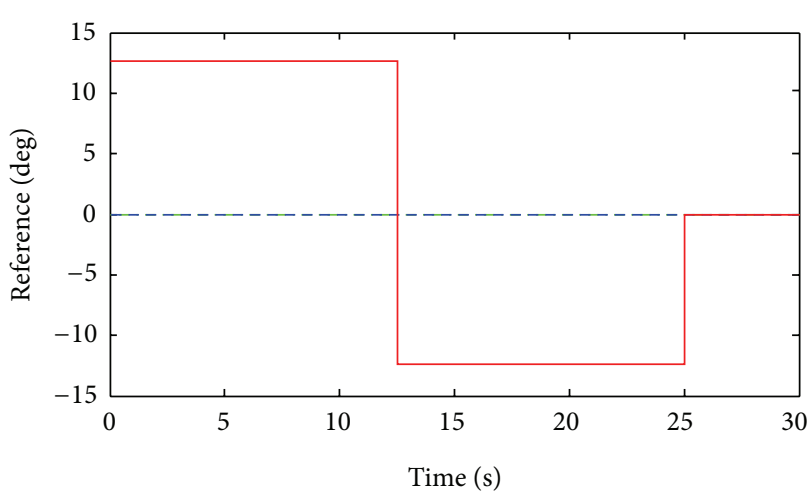

$$
\begin{array}{rr}
\cdots & \mu \\
--- & \alpha \\
- & \beta
\end{array}
$$

Figure 5: Reference.

by using the robust control toolbox). But the control inputs $\delta_{a}$ and $\delta_{r}$ actually exceed their amplitude limits as Figure 6(b) displays. Imposing amplitude limits at the aircraft's inputs in the simulation, the tracking performance deteriorates, as shown in Figure 7(a). Thus, AW compensation should be considered. Using Theorem 5 and taking the delayed saturation index $g_{d}=0.65$, the designing matrix is

$$
F=\left(\begin{array}{cccccc}
-1.6399 & 0.0327 & 0.6927 & -0.2444 & 241 & 3.8841 \\
0.0219 & -0.2281 & 0.0013 & 45.841 & 0.0003 & 38.787 \\
0.0155 & -0.2099 & 0.0010 & -10.303 & 0.1976 & 174.09
\end{array}\right) \text {, }
$$

and the obtained optimal $\mathscr{L}_{2}$ gain is $\gamma=$ 5.7294. The corresponding tracking performance is shown in Figure 8(a). The AW compensator designed in [14] which is based on immediate activation is further presented here for comparison. Solving LMI (13) in [14], the designing matrix is

$$
F=\left(\begin{array}{cccccc}
-731.13 & 16.222 & 425.51 & -106.50 & 98175 & 1634.7 \\
31.960 & -329.78 & 2.4337 & 54547 & -76.769 & 59246 \\
11.554 & -123.60 & 1.0543 & -5903.4 & 79.153 & 10750
\end{array}\right)
$$

and the optimal $\mathscr{L}_{2}$ gain is $\gamma=5.7298$. The corresponding tracking performance is shown in Figure 7(b). Our method is also compared with the delayed AW compensator proposed in [10]. Using the method of [10], the tracking performance is shown in Figure 8(b).

Comparing the tracking performance in Figure 8(a) with the uncompensated one as in Figure $7(\mathrm{a})$, fluctuation in $\mu$ channel decreases significantly in Figure 8(a). The AW compensator plays its role. From Figures 8(a) and 7(b) which are based on the immediate AW compensation, the output dynamic property in Figure 8(a) is preferable. This is because modest saturation can be seen as a small amount of plant uncertainty, and since the nominal controller is robust, the system still demonstrates good performance during this modest saturation stage. Immediate AW compensation may destroy this equilibrium. From Figures 8(a) and 8(b) with the static delayed AW compensator, the output performance in Figure $8(\mathrm{a})$ is no worse. This is because the AW compensator 

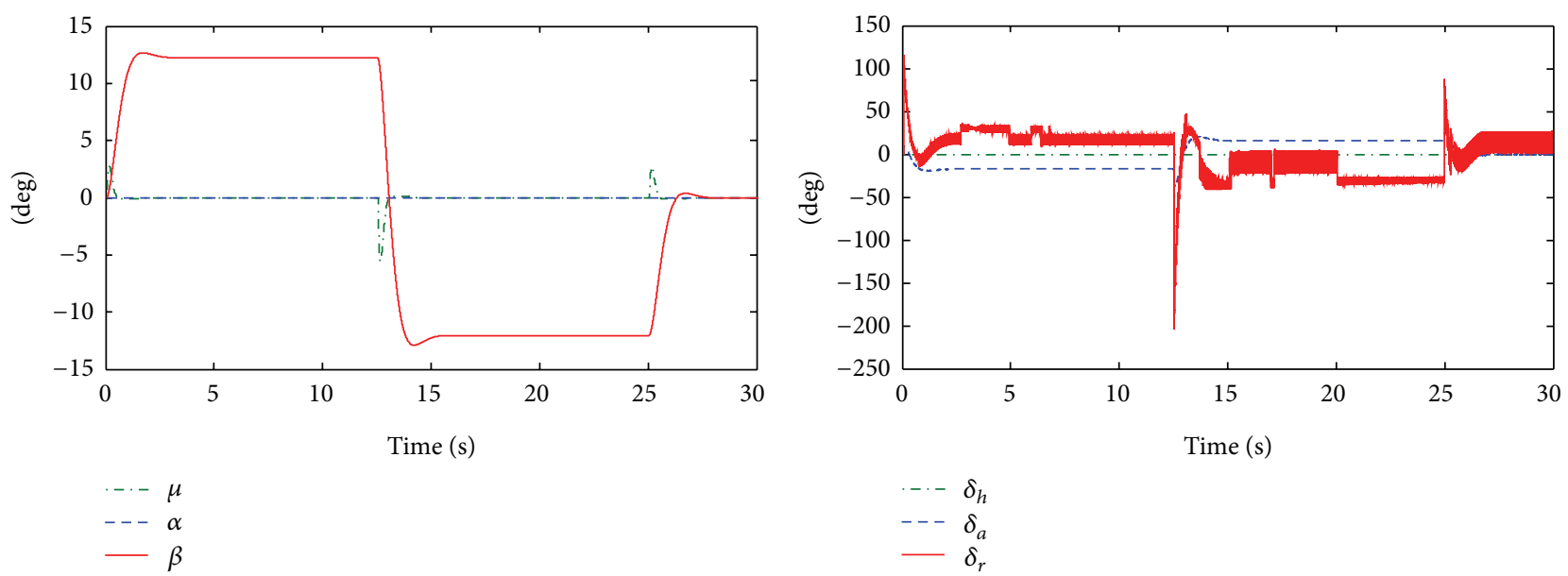

(a) Output response

(b) Control inputs

FIGURE 6: Linear tracking response with pulse reference in $\beta$ (no saturation).
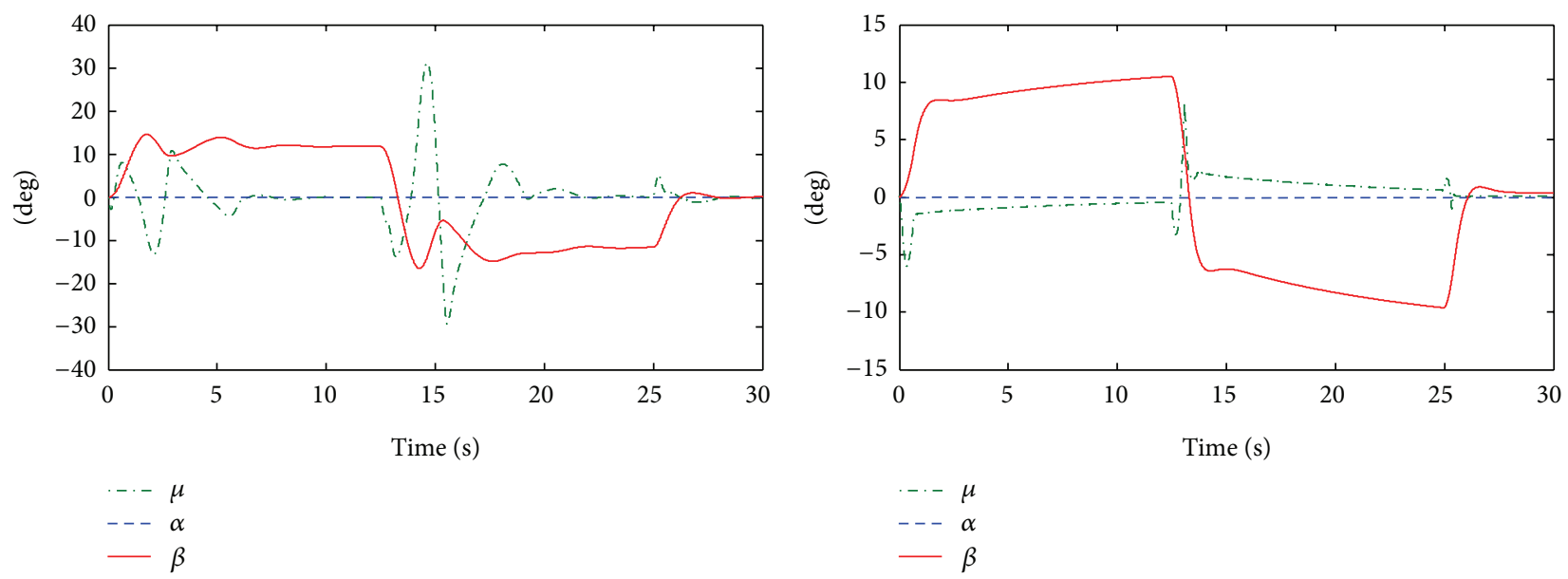

(a) Without AW compensator

(b) With AW compensator

FIGURE 7: Saturated tracking response with pulse reference in $\beta$ (with saturation).

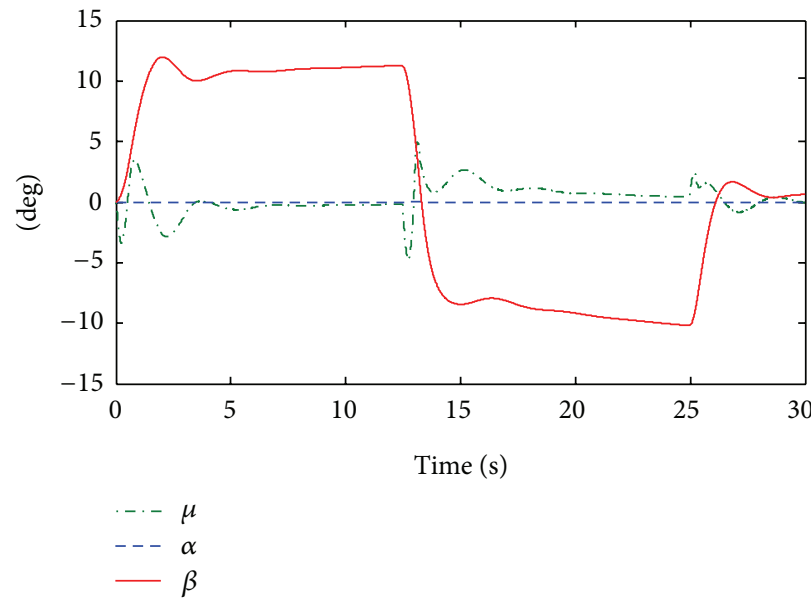

(a) Delayed AW compensator

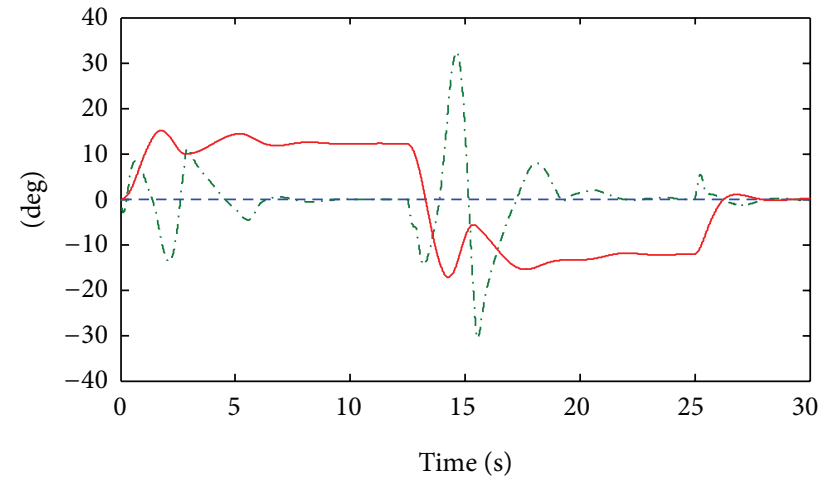

$$
\begin{array}{r}
-\cdots \\
--\alpha \\
-\beta
\end{array}
$$

(b) Delayed AW compensator proposed in [10]

FIGURE 8: Saturated tracking response with delayed AW compensator. 
is a plant-order dynamic one, while the delayed AW compensator proposed in [10] is static.

\section{Conclusion}

In this paper, the AW control problem based on the decoupling structure proposed by Weston and Postlewaite is investigated. Based on the decoupling AW structure, matrix $F$ is to be designed to determine $M(s)$, which is a right coprime factor of the plant $G(s)$. Motivated by the novel idea of delaying the activation of the AW compensator, a new decoupling structure with delayed activation of AW compensator is developed. A time-varying gain $G_{d}$ is introduced, and $\widehat{G}(s)=G(s) G_{d}$ is treated as a new quasi-LPV plant to deal with the delayed AW control problem. For both single-input and multi-input plants, a set of small scale LMIs are derived to calculate the designing matrix $F$ for AW compensator. Only simple LMIs are needed to be solved, while the LMIs in [14] to be solved are large. Most of all, the AW technique proposed here has a better performance applied in the application example than in [14] and has no worse performance than [10].

\section{Acknowledgments}

This work is jointly supported by National Science Foundation of China under Grants 60974078 and 90916003 and Foundation of National Defense B0320110023.

\section{References}

[1] K. Peng, H. S. Su, and Y. P. Yang, "Coordinated control of multi-agent systems with a varying-velocity leader and input saturation," Communications in Theoretical Physics, vol. 52, no. 3, pp. 449-456, 2009.

[2] M. V. Kothare, P. J. Campo, M. Morari, and C. N. Nett, "A unified framework for the study of anti-windup designs," Automatica, vol. 30, no. 12, pp. 1869-1883, 1994.

[3] S. Tarbouriech and M. Turner, "Anti-windup design: an overview of some recent advances and open problems," IET Control Theory \& Applications, vol. 3, no. 1, pp. 1-19, 2009.

[4] E. F. Mulder, M. V. Kothare, and M. Morari, "Multivariable antiwindup controller synthesis using linear matrix inequalities," Automatica, vol. 37, no. 9, pp. 1407-1416, 2001.

[5] G. Grimm, J. Hatfield, I. Postlethwaite, A. R. Teel, M. C. Turner, and L. Zaccarian, "Antiwindup for stable linear systems with input saturation: an LMI-based synthesis," IEEE Transactions on Automatic Control, vol. 48, no. 9, pp. 1509-1525, 2003.

[6] J. M. Gomes da Silva Jr. and S. Tarbouriech, "Antiwindup design with guaranteed regions of stability: an LMI-based approach," IEEE Transactions on Automatic Control, vol. 50, no. 1, pp. 106111, 2005.

[7] L. Lu and Z. Lin, "A switching anti-windup design using multiple Lyapunov functions," IEEE Transactions on Automatic Control, vol. 55, no. 1, pp. 142-148, 2010.

[8] P. F. Weston and I. Postlethwaite, "Linear conditioning for systems containing saturating actuators," Automatica, vol. 36, no. 9, pp. 1347-1354, 2000.

[9] M. C. Turner and I. Postlethwaite, "A new perspective on static and low order anti-windup synthesis," International Journal of Control, vol. 77, no. 1, pp. 27-44, 2004.
[10] S. Sajjadi-Kia and F. Jabbari, "Modified anti-windup compensators for stable plants," IEEE Transactions on Automatic Control, vol. 54, no. 8, pp. 1934-1939, 2009.

[11] E. Prempain, M. C. Turner, and I. Postlethwaite, "Coprime factor based anti-windup synthesis for parameter-dependent systems," Systems \& Control Letters, vol. 58, no. 12, pp. 810-817, 2009.

[12] S. Sajjadi-Kia and F. Jabbari, "Modified anti-windup compensators for stable plants: dynamic anti-windup case," in Proceedings of the 48th IEEE Conference on Decision and Control Held Jointly with the 28th Chinese Control Conference (CDC/CCC '09), pp. 2795-2800, Shanghai, China, December 2009.

[13] J. S. Yee, J. L. Wang, and N. Sundararajan, "Robust sampleddata $H_{\infty}$-flight-controller design for high $\alpha$ stability-axis roll maneuver," Control Engineering Practice, vol. 8, no. 7, pp. 735747, 2000.

[14] M. C. Turner, G. Herrmann, and I. Postlethwaite, "Anti-windup compensation using a decoupling architecture," in Advanced Strategies in Control Systems with Input and Output Constraints, vol. 346 of Lecture Notes in Control and Information Sciences, pp. 121-171, Springer, Berlin, Germany, 2007. 


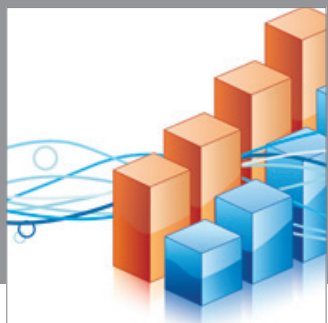

Advances in

Operations Research

mansans

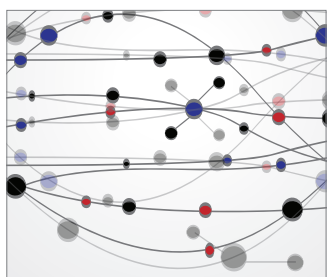

The Scientific World Journal
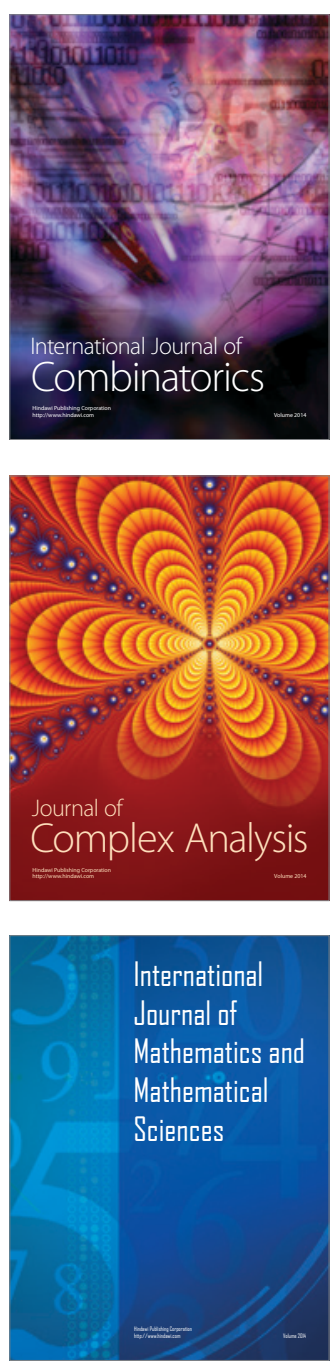
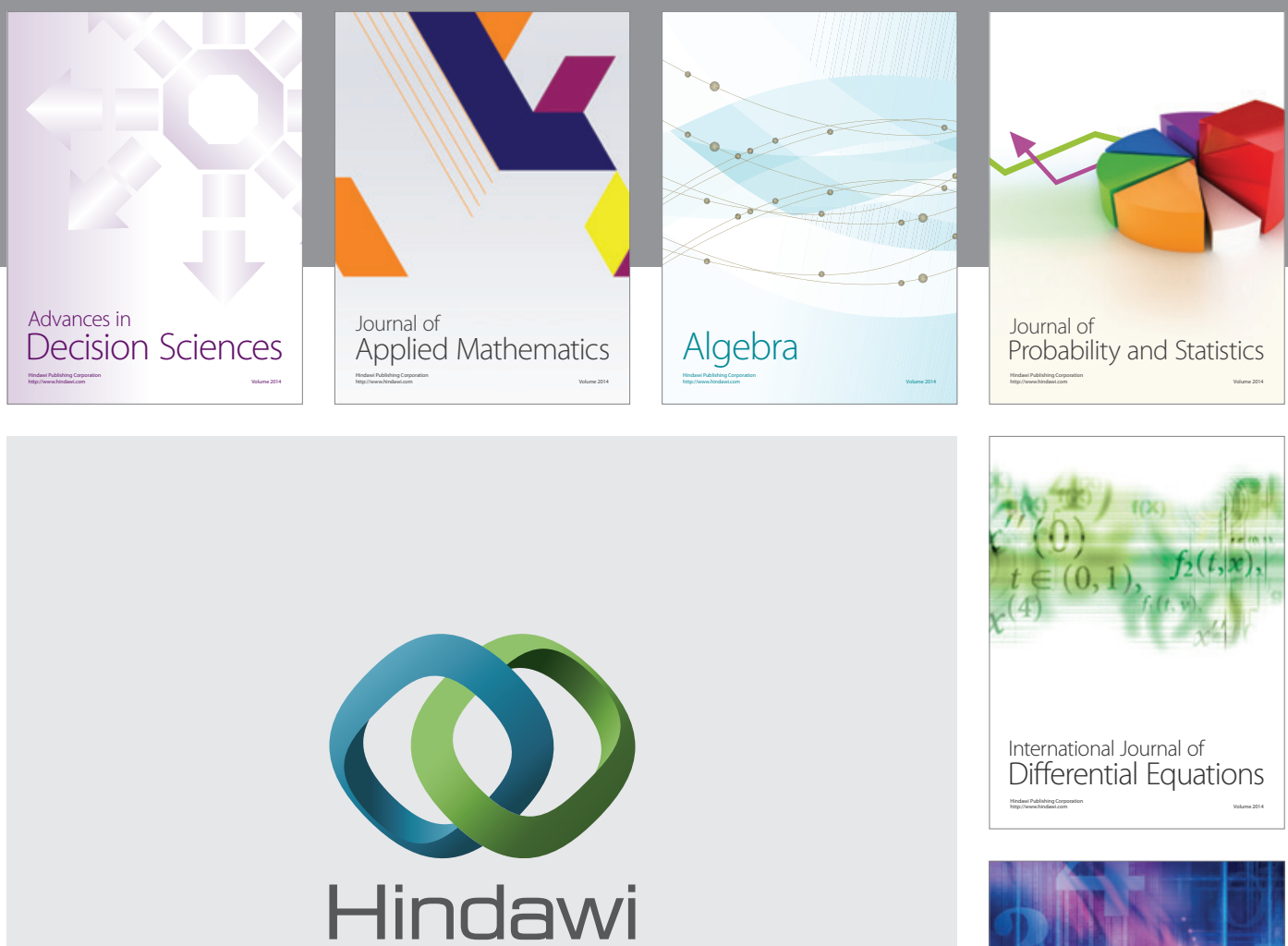

Submit your manuscripts at http://www.hindawi.com
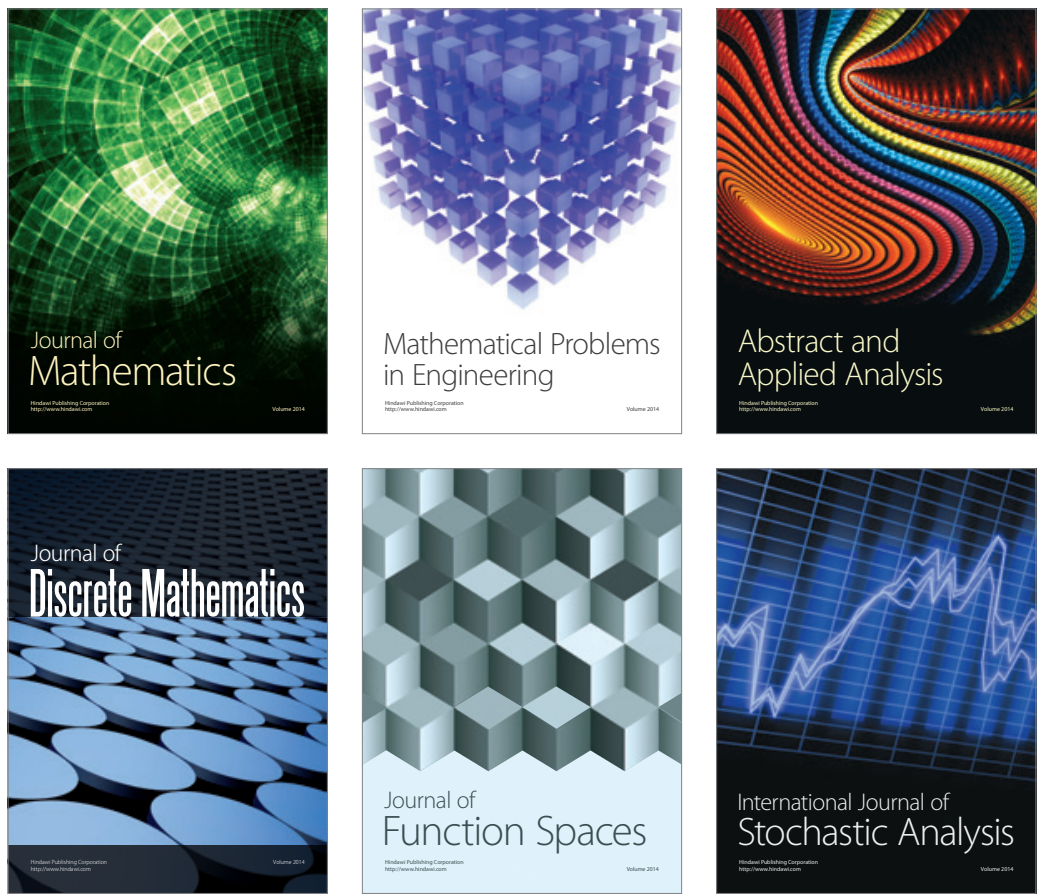

Journal of

Function Spaces

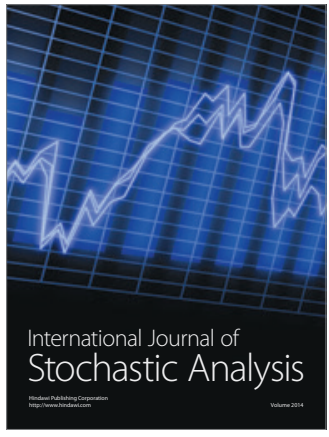

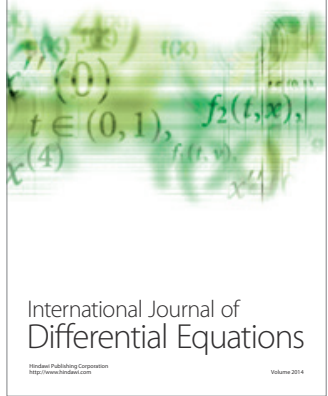
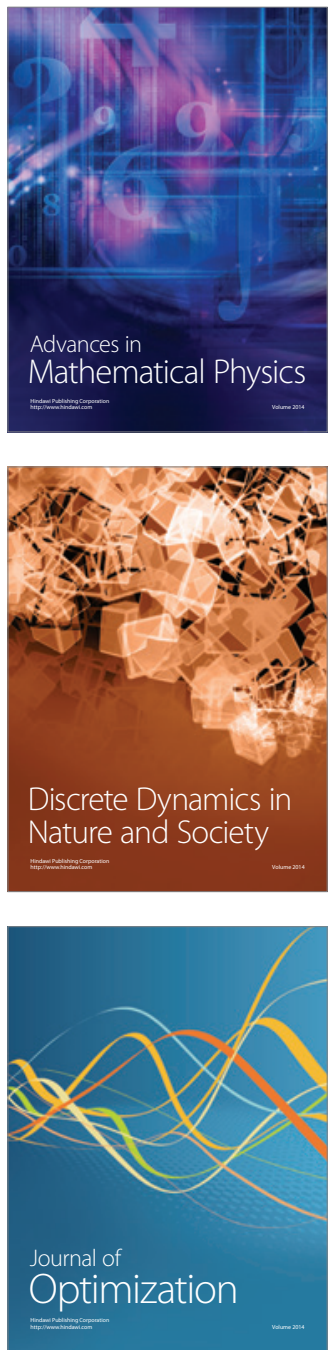